\section{Beyond Massive MIMO: living at the interface of electromagnetics and information theory}

Professor Michalis Matthaiou

Centre for Wireless Innovation, Queen's University Belfast,

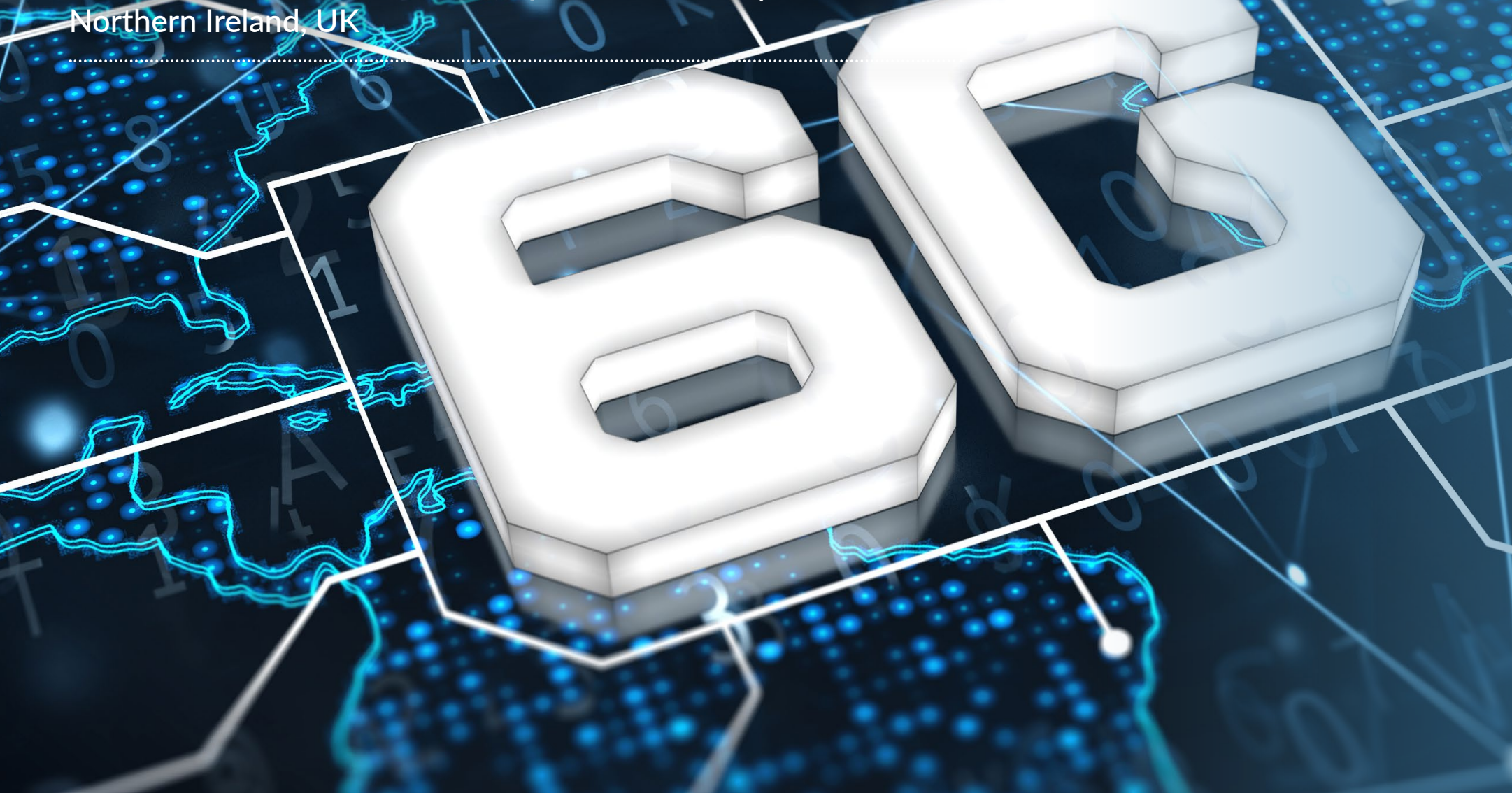

The long-awaited rendezvous of Shannon and Maxwell.

"Thoroughly conscious ignorance is the prelude to every real advance in science."

James Clerk Maxwell (1831-1879).
Exactly 190 years since the birth of James Clerk 19 VIl, who is widely regarded as the father of electromagnetism, the term way to describe the world of wireless way to describe the world of wireless
communications. In the context of wireless policy, 'radio waves' is shorthand for a portion of the broader spectrum of electromagnetic (EM) radiation. In physics, EM radiation is defined in a unified way a self-propagating transverse oscillating wave of synchronised electric and magnetic fields.

More recently, in the late 1940 s, Claude Elwood Shannon (19162001) harnessed the skills gained in the field of cryptanalysis for national defence during World War II to develop his groundbreaking information theory (Shannon, 1948) a mathematically tractable framework for the quantification, storage, and communication of digital information.

Whilstinformation theory has served the wireless communications community particularly well for 70 years; it is a - Statistical tool based on mathematica logie, not the laws of physics. For example, it does not consider the electromagnetic propagation of conminunications signals as governed by

Classical information theory now needs to be extended and reshaped to incorporate the main feature of future communication systems, namely their capability of sensing the system's response to the radio waves, (t) modification. for PEATRICE project is the unification Ff infor prom theory and EM theory to chacterse 'wiress waves' to to che 'wieless waves' to expand the frontiers of wireless exercise: information theory is based on probabilistic tools, whis electromagnetic theory encompasses Maxwell s wave equations. As such, the interface between these two theories inherently a challenging landscape.
The massive MIMO paradigm Among various technological advances that have been incorporated in $5 \mathrm{G}$ standards compared to $4 \mathrm{G}$, the key is multiple-output (MaMi) MaMi was originally proposed as a new academi concept in 2010 in a breakthrough paper from Marzetta (2010). Barely ten years later, MaMi is being taken up across the telecom space, from academic researchers to senior industrial managers and policymakers (Release 15). With $\mathrm{MaMi}$, we refer to cellular systems that deploy an unconventionally large number of antennas (hundreds or even thousands) at base stations (BSs) to serve dozens or even hundreds of users.

The importance of MaMi stems from the extra degrees of freedom it offers, thanks to an excessive number of antennas. It reception/transmit beamforming, gives nearly orthogonal user channels, and provides resilience to imperfect channel knowledge and hardware imperfections.

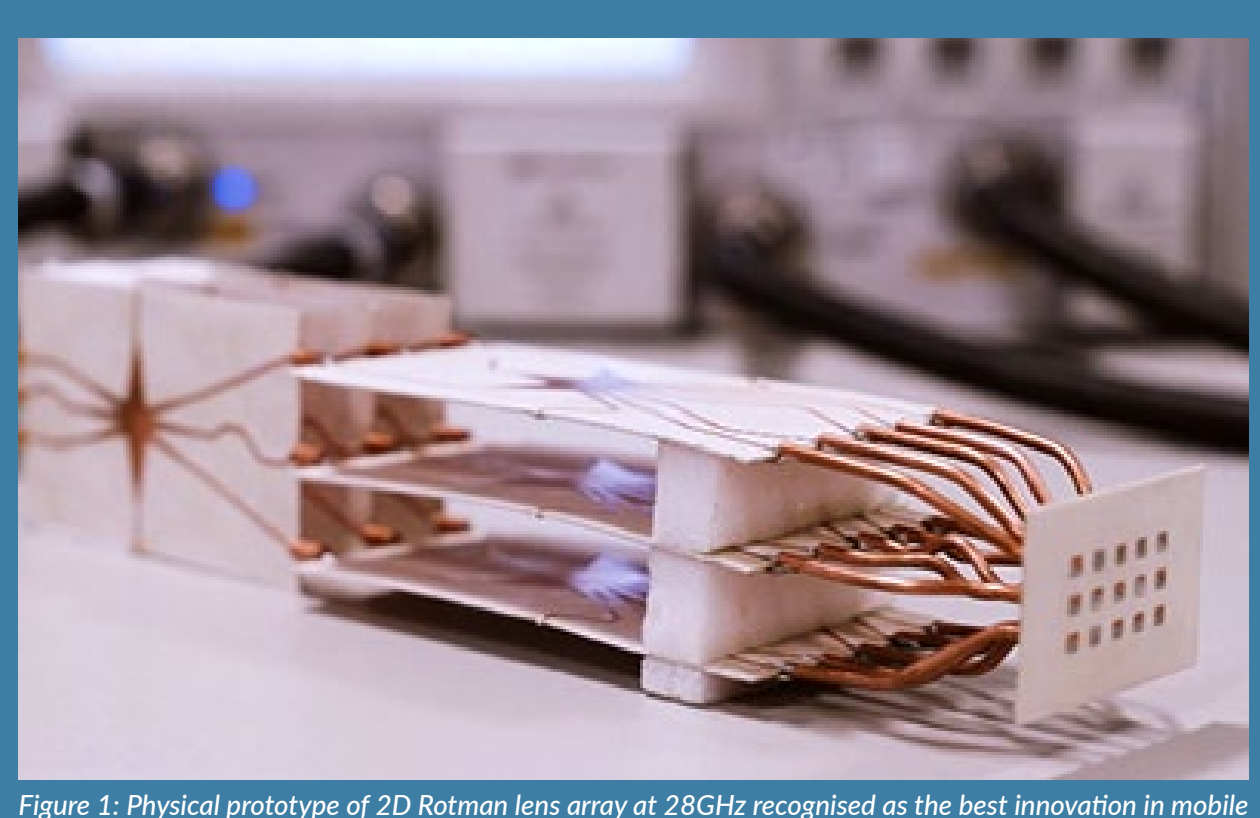

Figure 1: Physical protot

The fundamental concept behind the BEATRICE project is the unification of information theory and EM theory to characterise 'wireless waves' to expand the frontiers of wireless communications. 


\section{PROJECT NAME}

\section{BEATRICE}

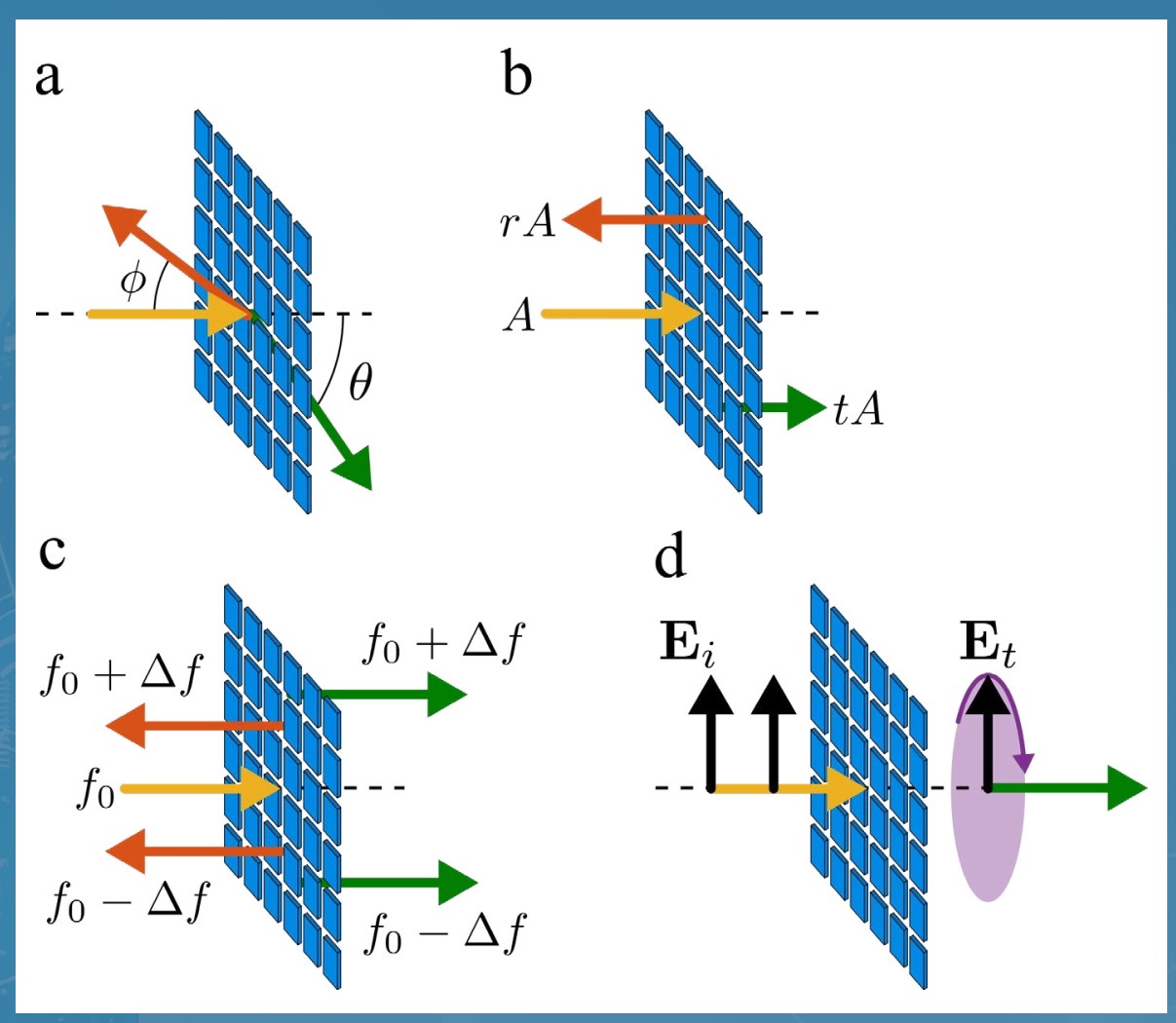

Figure 4: (a) Ang
modulation.

More specifically, the recently proposed framework to cover angle, amplitude, spatio-temporal coding (Cui et al., 2014) frequency and polarisation modulation spatio-temporal coding (Cui et al., 2014) by means of intelligent metasurfaces will
lead to a breakthrough in the way we lead to a breakthrough in the way we
encode/decode information in future encode/decode information in future Finally, we will physically synthesise and wireless networks as it will enable the highly desirable 'recycling' of radio waves. Transforming the breakthrough
idea of intelligent metasurfaces to a idea of intelligent metasurfaces to a rigorous modulation technique is a very
challenging exercise and will be one of the main objectives of this WP. To this end, we will develop a theoretical validate the proposed solutions usin valide the proposed solutions using our extensive, world-class lab facilities QUB. These novel prototypes will research fields, such as electronics, elaborate on super-directive antenna
arrays, an old concept that has faced arrays, an old concept that has faced
criticism. Yet there is strong evidence that super-directive antennas will constitute a critical component of many future communication systems since they can offer extremely sharp energy focusing and much lower interference levels. For these reasons, it is essential to go back to first principles and develop solutions for their for cellular and wireless power transfer services. practical integration.

BEATRICE is going to develop a radical technological paradigm called resonant intelligent metasurfaces.

\section{PROJECT SUMMARY}

Information theory, proposed by Claude Shannon in 1948, has served the wireless communications community particularly well for seven decades. Looking ahead, this theoretical framework needs to be extended to account for the unique electromagnetic properties of concurrent (e.g. 5G) and future (e.g. beyond 5G, 6G) massive multiple-input multipleoutput systems. This is a challenging exercise: information theory is based on probabilistic tools whilst electromagnetic theory encompasses Maxwell's wave equations. BEATRICE aims to use these two theories in parallel to create new fundamental understanding, modulation techniques and physical prototypes. These findings will have a far-reaching impact on cellular communications, wireless power transfer, radar, and optical wireless communications.

\section{PROJECT LEAD}

Michalis Matthaiou is Professor of Communications Engineering and Signal for Wireless Inneputy Director of the Centre Belfast. Under his co-leadership, CWI has become one of the world's leading research and innovation centres in wireless communications systems. Michalis' seminal research contributions have underpinned the development of $5 \mathrm{G} / 6 \mathrm{C}$ wireless technologies, with his breakthroug work on massive MMMO removing major scepticism about this technology. He is the us prestigious accolades to date, including a 2019 EURASIP Early Career Award and a 2018-2019 RAEng/Leverhulme Trust Senior Research Fellowship.

\section{CONTACT DETAILS}

Professor Michalis Matthaiou

ECIT Institute, Queen's University Belfast, Queen's Road, Queen's Island, Catalyst Inc. Belfast, BT3 9DT, Northern Ireland, UK

\section{III +44 2890971789}

m.matthaiou@qub.ac.uk

https://sites.google.com/site/

\section{References}

Cui, T., Qi, M., Wan, X., Zhao, J. and Cheng, Q. (2014) Coding metamaterials, digital metamaterials
and programmable metamaterials,' Light: Science and Applications, 3(e218). doi: 10.1038/sa.2014.99. Marzetta T.L. (2010) 'Noncooperative cellular wireless with unlimited numbers of base station

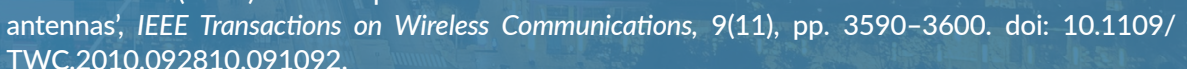

Shannon C.E. (1948) 'A mathematical theory of communication', Bell System Technical Journal, 27
pp. $379-423,623-656$ doi: $10.1002 / 1.1538-7305$, 1948 th01338. robust communication by harnessing knowledge about the EM properties propagation medium.

\section{https://scholar.google.se} citations?user=g6HXpqoAAAAJ\&hl=en

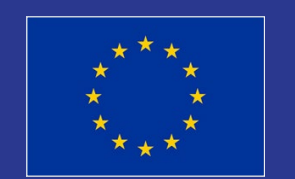

FUNDING

This project has received funding from the European
Union's Horizon 2020 research and innovation
programme under grant agreement No. 101001331 . 